\title{
Bildungsreformen in Deutschland
}

\section{Philipp Gonon}

Online publiziet: 28. April 2015

(c) Die Autor(en) 2015. Dieser Artikel ist auf Springerlink.com mit Open Access verfügbar.

\section{Rezension zu:}

Ullrich Bauer, Axel Bolder, Helmut Bremer, Rolf Dobischat \& Günter Kutscha (Hg.) (2014). Expansive Bildungspolitik - Expansive Bildung? Springer VS, Wiesbaden, 451 Seiten, 58,31 €, ISBN 978-3-658-06669-7

Im Zentrum des Sammelbandes steht die Frage nach den Bildungsreformen in der Bundesrepublik Deutschland, im Besonderen nach ihren Auswirkungen auf Selektion und subjektiven Lernbereitschaften.

Ingrid Lisop thematisiert bildungspolitische Paradoxien, die, wie sie anhand der bundesrepublikanischen Bildungsreform darlegt, zu „Switch-Politiken“, das heißt Ersatzhandlungen führen, welche nach wie vor Bildungsverlierer produzieren. Die Frage nach Gerechtigkeit im Rahmen von Bildungsreformen greift auch Jürgen Gerdes auf. Teilhabe- und Chancengerechtigkeit seien heute auf die Frage der sozialen Mobilität und auf ,eigenverantwortliche“, individuelle Kompetenzentwicklung reduziert worden. Bettina Kohlrausch unterscheidet als Zielsetzungen des investiven Sozialstaats Arbeitsmarktintegration, Statuserhalt sowie Ermöglichung selbstbestimmter Lebensführung. Wie sie anhand einer Evaluation von Reformprojekten zur Dualisierung des Schulalltags zwecks Steigerung der Übertrittsquote von Hauptschülern in die Arbeitswelt darlegt, steht die durchaus auch Erfolge aufweisende Arbeitsmarktintegration einer potentiell benachteiligten Gruppe so stark im Vordergrund, dass andere Zielsetzungen der gesellschaftlichen Teilhabe und selbstbestimmten Lebensführung ausgeblendet bleiben.

Prof. Dr. P. Gonon $(\bowtie)$

Institut für Erziehungswissenschaft der Universität Zürich,

Universität Zürich, Kantonsschulstrasse 3,

8001 Zürich, Schweiz

E-Mail: gonon@ife.uzh.ch 
Einem anderen Segment, nämlich dem Verbleib von Hochschulabsolventinnen und -absolventen und der schwankenden Nachfrage nach Fachkräften widmen sich die empiriegestützten Ausführungen von Andrä Wolter. Die Berufsperspektiven und Beschäftigungschancen dieser Gruppe werden nach wie vor als intakt betrachtet angesichts steigender Qualifikationsanforderungen. Allerdings führe die weiter wachsende Anzahl der Hochschulabgänger zu einer Zunahme von Übergangsproblemen und Beschäftigungsrisiken. Auch der Beitrag von Robert Helmrich und Gerd Zika thematisiert das Wechselspiel von Fachkräftemangel und Akademisierung mit einem besonderen Fokus auf den Ingenieur. Die Zunahme von Ingenieuren aus dem Bereich der Hochschulen gehe einher mit einem Rückgang der Auszubildendenzahlen, was zur Folge habe, dass Ingenieure künftig auch weniger anforderungsreiche Tätigkeiten ausführen müssten.

Umgekehrte Risiken, nämlich Ausgrenzung aus Arbeitsmarkt und Gesellschaft, zeichnen sich für Geringqualifizierte ab. Die Autoren Olaf Groh-Samberg und Henning Lohmann fokussieren hierbei die Rolle der Bildung, vermeiden aber den nur schwer fassbaren Begriff „Bildungsarmut“. Sie unterscheiden vielmehr eine Ausgrenzung vom Arbeitsmarkt, die stark vom Fehlen einer beruflichen Ausbildung abhänge, von einer sozialen, politischen und kulturellen (Des-)Integration, die im Zusammenhang mit der allgemeinen Schulbildung zu sehen ist. Im Zuge der Bildungsexpansion finde eine überproportionale Zunahme von Ausgrenzungsrisiken statt, die auch zu einer weiteren De-Feminisierung und Ethnisierung der Geringqualifizierten führen würden.

Wulf Hopf erörtert den Wandel des Regimes sozialer Selektion im Bezug zur Bildungsexpansion. Der Autor diskutiert die stetig im Raum stehende Frage, ob von einem bildungs- und berechtigungsbezogenem „Upgrading“ alle Schichten gleichermaßen oder nur bestimmte mehr oder weniger profitiert hätten, ob also der von Ulrich Beck so bezeichnete „Fahrstuhleffekt" keine oder doch eine relative Annäherung der Bildungschancen ergeben hätte und wie sich dieses allenfalls auf das „Ungleichheitsbewusstsein“ der Subjekte auswirke. Es sei gerade die Gleichzeitigkeit unterschiedlicher Prozesse, wie beispielsweise die Schrumpfung von Hauptschulen, die „Abwahl“ von Schulen in Brennpunkten oder die Neigung von Eltern aus höheren Schichten, ihre Kinder auf Privatschulen zu schicken, die eindeutige Befunde zur sozialen Integrationsleistung des Bildungssystems erschweren. Auch Michael Vester geht der Frage nach, inwiefern sich die aus seiner Sicht weiterhin bestehende ständische Stufung der Bildungs- und Berufswege reproduziert. Das Sortierungssystem habe sich den neuen Gegebenheiten, wie sie sich durch Reformen ergeben haben, angepasst und würde z. B. in das Berufsbildungssystem selbst eine Zweiteilung entlang ständisch-kultureller, geschlechtlicher und ethnischer Linien hineintragen, die auch trotz insgesamt erhöhter Bildungsbeteiligung bestehen.

Alfred Scherr geht der Frage der (Un-)Wahrscheinlichkeit von Bildungsaufstiegen nach, gerade auch mit kritischem Blick auf „rational choice“ und klassen- sowie milieuspezifische Erklärungsmuster, deren (sozial-)deterministische Grundannahmen durch empirische Forschungen zu irritieren seien. Eine konkrete Fallstudie - nämlich die Passungskonstellationen hinsichtlich des Zugangs zu exklusiven gymnasialen Bildungseinrichtungen - bildet den Hintergrund einer von Werner Helsper und Koautoren verfassten Analyse des Zusammenspiels von milieuspezifischen 
Erwartungen und Schulen, die sich darauf einstellen müssen, bzw. Familien, die auch mit entsprechenden Bildungsansprüchen konfrontiert werden.

Rosa Bracker und Peter Faulstich greifen in ihrem Beitrag die Frage der Weiterbildungsbeteiligung auf. In der Weiterbildungsbeteiligungsforschung, die sie kurz darstellen, ist eine Verlagerung vom vorwiegend strukturell geprägten Bedingtheitsdiskurs hin zu einem stärker subjektive Begründungen auslotenden Ansatz, welcher Selektivität konstituiert, auszumachen. Während aus subjektiver Sicht Lernschranken - ähnlich alltagspraktischen Problemen - als lösbar betrachtet werden, sind demgegenüber Lernhemmnisse biographisch fest verankerte und damit nicht einfach zu verändernde individuelle Voraussetzungen, die auf ein institutionalisiertes Lernen angewiesen sind, um Handlungsspielräume zu erweitern.

Schlussfolgernd lässt sich festhalten, dass es der Herausgeberschaft gelungen ist, eine interessante Melange von Studien und Überlegungen, zumeist gestützt auf neuere Untersuchungen zu den verschiedenen Bereichen des Bildungssystems zusammenzuführen, die eine kritische Bilanz der Bildungsreformen in der Bundesrepublik Deutschland nahelegen. Vor allem der Blick auf die Benachteiligten und auf Ausgrenzungsmechanismen, bzw. auch auf Hindernisse bezüglich Bildungsbeteiligung ist bedeutsam, um den „Erfolg“ der Bildungsexpansion einzuschätzen. Kritisch ist anzumerken, dass bezüglich Bildungsreformen viele Fragen nicht thematisiert oder vertieft werden. Weder der titelgebende Begriff „expansive“ Bildungspolitik noch das Konzept „expansive Bildung“ werden im Band näher erörtert. Die Herausgeber versuchen immerhin in der Einleitung, die verschiedenen Ausführungen thematisch $\mathrm{zu}$ ordnen und diese auch historisch zu verorten, jedoch wirken einige Beiträge etwas verloren. Dennoch ermöglicht der Band eine auf aktuelle Forschung beruhende kritische Bestandsaufnahme der Bildungsreformen der letzten Jahrzehnte.

Open Access Dieser Artikel wird unter der Creative Commons Namensnennung 4.0 International Lizenz (http://creativecommons.org/licenses/by/4.0/deed.de) veröffentlicht, welche die uneingeschränkte Nutzung, Verbreitung und Wiedergabe für beliebige Zwecke erlaubt, sofern Sie den/die ursprünglichen Autor(en) und die Quelle ordnungsgemäß nennen, einen Link zur Creative Commons Lizenz beifügen und angeben, ob Änderungen vorgenommen wurden. 